\title{
Inductor Compensation in Three Phase PFC Control with Decoupling the Input Voltage and Bus Voltage
}

\author{
Guang Luo ${ }^{1, a}$, Wu Ai ${ }^{1}$, Bing Chen ${ }^{1}$ and Yi Liu ${ }^{1}$ \\ ${ }^{1}$ Huazhong University of Science and Technology, School of Mechanical Science and Engineering, 430074 Wuhan , \\ China
}

\begin{abstract}
As the nonlinear structure of the boost ac/dc topology, the PFC is difficult to be controlled. The average state space theory has revealed that the choke current is determined by duty-ratio coupled with input voltage and bus voltage, which makes the high-performance sinewave current track control more challenging. To remove disturbing variables and reduce the zero-crossing distortion, the decoupling control strategy is presented. Also, it is shown in this paper that the variable inductance has a great influence on PFC and THDI. As the inductor in boost converter varies with the current, a compensating coefficient of current is also proposed. The decoupling strategy and the inductor compensation strategy are implemented on a UPS with three phase input voltage. Numerical simulation and experimental results has indicated the high-performance of these control strategy.
\end{abstract}

\section{Introduction}

As a non-isolate ac/dc PFC converter, the boost converter is widely used in various industrial applications due to its simple circuit structure, high reliability, low cost and low current ripple in high frequency. Among the three current modes, CCM, DCM and BCM, CCM achieves the highest occupation ratio in medium and high power field [1]. In general, the sinusoidal wave generator is used for providing the set value of inner current loop. Its realization algorithm includes looking up table and following mains supply. Generally, the former is able to offer a better performance in terms of total harmonic distortion (THD), while the latter is able to offer a better performance in terms of power factor (PF). Besides, the look-up table needs a phase locked loop (PLL) accurately detect the zero crossing of sinusoidal wave, which will increase the complexity of the control system and load of the CPU [2].

The conventional analysis method is using the average state space theory under high-frequency carrier, and establishing controlled object [3-4]. When the input voltage is very low, boost IGBT works at an almost $100 \%$ duty ratio, which will lead to the phase lead of input current and thus results in zero distortion, especially under high ac line frequencies [5]. Therefore, the merely conventional PID correction is unable to reduce the effect of the leading phase of input current.

So, many new control technique have been proposed. In [6], a feedforward current control approach is proposed for a boost PFC converter. In this paper, an additional feedforward signal is shown to further reduce input current harmonic distortion, especially near the zero crossing of the

\footnotetext{
${ }^{a}$ Corresponding author : andylaw_lg@qq.mail 
input voltage. In [7], a duty-ratio feedforward control approach is proposed. Essentially, both of the control techniques add an open-loop control method to the closed-loop control method. In [8], a new current detect method is proposed, but it will aggravate processing burden. In [9-11], various kinds of topology is proposed, but additional hardware may increase the cost.

In this paper, a decoupling control strategy is proposed on the basis of the conventional doubleloop control strategy. After decoupling the input voltage and bus voltage, the input current is completely determined by duty-ratio. Then, the segment from duty-ratio to input current can be considered as a pure integrator model. Due to the pure integrator, a compensating coefficient could exactly offset the fluctuation of inductance value which plays a great role in the control.

\section{The Small Signal Model of The Boost Topology}

The averaged dynamics of the PFC over one switching cycle is given by

$$
\left\{\begin{array}{c}
\frac{d i_{L}}{d t}=\frac{-(1-d(t))}{L} V_{B U S}(t)+\frac{V_{\text {in }}(t)}{L} \\
\frac{d V_{B U S}(t)}{d t}=\frac{(1-d(t)) i_{L}(t)}{C}-\frac{V_{B U S}(t)}{C Z}
\end{array} .\right.
$$

The average value and its fluctuation is given by

$$
\left\{\begin{array}{l}
d(t)=D+\hat{d} \\
i_{L}(t)=i_{L}+\hat{i}_{L} \\
V_{B U S}(t)=V_{B U S}+\hat{V}_{B U S} \\
V_{i n}(t)=V_{i n}+\hat{V}_{i n}
\end{array}\right.
$$

Thus, the balance equation and the small signal linear equation can be deduced as follows:

$$
\begin{gathered}
\left\{\begin{array}{c}
V_{\text {in }}=(1-D) \cdot V_{B U S} \\
(1-D) \cdot i_{L}=V_{B U S} / Z
\end{array}\right. \\
\left\{\begin{array}{l}
\frac{d \hat{i}_{L}}{d t}=-\frac{1-D}{L} \hat{V}_{B U S}+\frac{\hat{d}}{L} V_{P B U S}+\frac{\hat{V}_{i n}}{L} \\
\frac{d \hat{V}_{B U S}}{d t}=\frac{1-D}{C} \hat{i}_{L}-\frac{\hat{d}}{C} i_{L}-\frac{\hat{V}_{B U S}}{C Z}
\end{array} .\right.
\end{gathered}
$$

Deduced from these equations, an inner current controlled object from $\hat{d}$ to $\hat{i}_{L}$ is written as

$$
\begin{aligned}
& \frac{\hat{i_{L}}(\mathrm{~s})}{\hat{d}(s)}=G_{d}\left(1+\frac{s}{\omega_{Z}}\right) /\left(\left(\frac{s}{\omega_{0}}\right)^{2}+\frac{s}{Q \cdot \omega_{0}}+1\right) \\
& G_{d}=2 V_{B U S} / Z(1-D)^{2}, \omega_{0}=(1-D) / \sqrt{L C}, \\
& \omega_{Z}=2 / C Z, Q=Z(1-D) \sqrt{C / L} .
\end{aligned}
$$

And a outer voltage controlled object from $\hat{i}_{L}$ to $\hat{V}_{B U S}$ is written as

$$
\frac{\hat{V}_{B U S}(\mathrm{~s})}{\hat{i}_{L}(s)}=\left((1-D)^{2} Z-L s\right) /((C Z s+2)(1-D)) \text {. }
$$

\section{The Control Stratagy}




\section{1 Close Loop Control Strategy}

The conventional control method is to establish the double loop model using PID regulator based on the two controlled objects as following (5), (6).

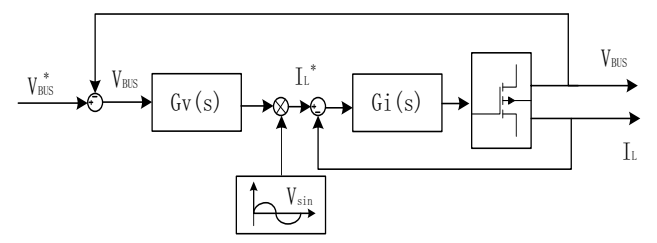

Figure 1. Conventional control block diagram

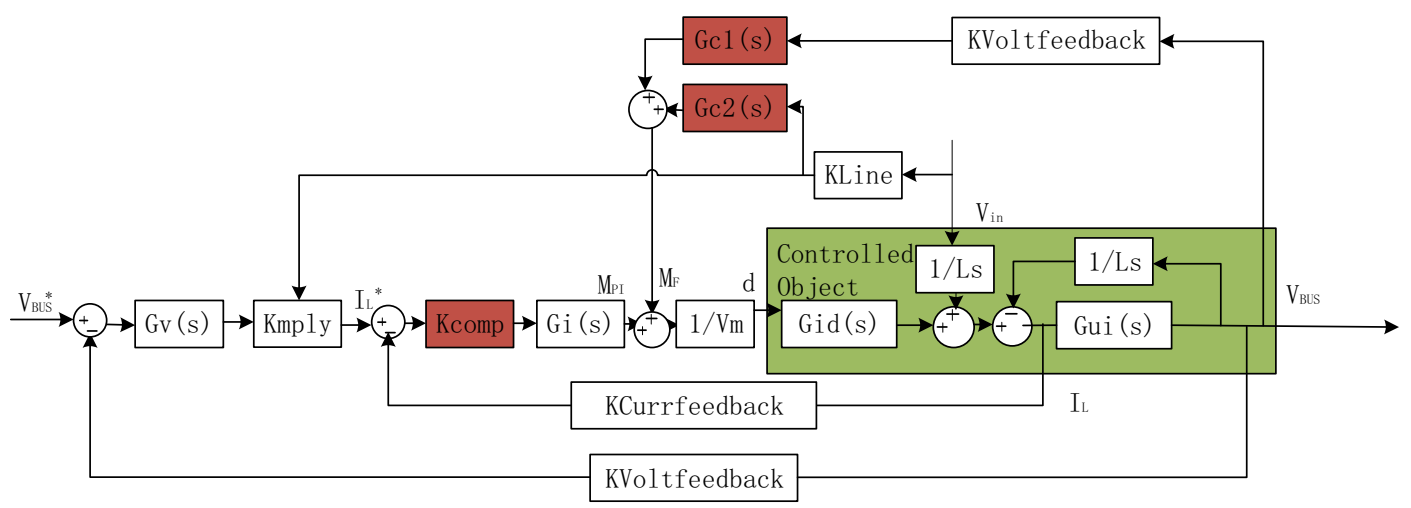

Figure 2.Advanced control block diagram

The voltage loop bandwidth is desired under $20 \mathrm{~Hz}$ to ensure the linear modulation of the $50 \mathrm{~Hz}$ sine multiplier, while the current loop bandwidth is desired much higher to ensure a fast tracking of sinusoidal input reference signals.

In conventional algorithm analysis under high frequency carrier, equation (5) is simplified as a simply pure integral model, and equation (6) is simplified as an inertial model. In fact, however, they are not.

According to analysis of bode graph, the high-performance compensating controllers are designed.

\subsection{Proposed Decoupling Strategy}

The controlled model of the boost PFC converter given by (1), represents a nonlinear system owing to the coupling of input duty ratio $d(t)$ with $V_{i n}(t)$ and $V_{B U S}(t)$. But the conventional controlled objects (5) and (6) are established without taking input voltage $V_{\text {in }}(t)$ and bus voltage $V_{B U S}(t)$ into consideration. In order to establish a linear system, a decoupling strategy is proposed.

Table 1. Control Parameters

According to (1),

\begin{tabular}{cc}
\hline \hline Name & Value \\
\hline Vm & 3906 \\
KLine & 10 \\
KVoltfeedback & 10 \\
KCurrfeedback & 100 \\
Kmply & 220 \\
\hline \hline
\end{tabular}




$$
\frac{d i_{L}}{d t}=\frac{d(t)}{L} V_{B U S}(t)+\frac{V_{i n}(t)}{L}-\frac{V_{B U S}(t)}{L}
$$

Under the high frequency, the change rate of $d(t)$ is much higher than that of $V_{B U S}(t)$.So, the $V_{B U S}(t)$ in $\frac{d(t)}{L} V_{B U S}(t)$ can be regarded as bus voltage set value $V_{B U S}^{*}$.

$$
i_{L}(s)=\operatorname{Gid}(s) * d(s)+\frac{V_{\text {in }}(s)}{L \mathrm{~s}}-\frac{V_{B U S}(s)}{L s}
$$

where

$$
\operatorname{Gid}(s)=\frac{V_{B U S}^{*}}{L s}
$$

As is shown in Fig.2, Gc1 and $G c 2$ are respectively presented to offset the voltage disturbance, and dc bus voltage disturbance.

Then,

$$
G c 1 * K V o l t f e e d b a c k * \frac{1}{V m} * \text { Gid }-\frac{1}{L s}=0
$$

And,

$$
G c 2 * \text { KLine } * \frac{1}{V m} * \text { Gid }+\frac{1}{L s}=0
$$

So,

$$
\left\{\begin{array}{l}
G c 1=V_{B U S}^{*} /\left(V m^{*} \text { KVoltfeedback }\right) \\
G c 2=-V_{B U S}^{*} /(V m * \text { Kline })
\end{array}\right.
$$

After decoupling the input voltage and bus voltage, equation (8) can be simplified to

$$
i_{L}(s)=\operatorname{Gid}(s) * d(s) \text {. }
$$

In contrast with equation (5), the inner current model is much briefer, owe to the decoupling strategy.

\subsection{Proposed Inductor Compensation}

According to hysteresis loop, the higher current is, the less the inductor value will become. The attenuation of the inductance value will make the inductor current rise sharply when IGBT is off, which will increasing current ripple in switch frequency when it is peak. Thus, the THDI will get worse. To achieve a high performance and a precise control of the current loop, the inductance value should be considered as constant. Therefore, the coefficient Kcomp is needed.

In the experience, Fe-Si is selected for the inductance core. The character graphs of the inductance is expressed in Fig.3.

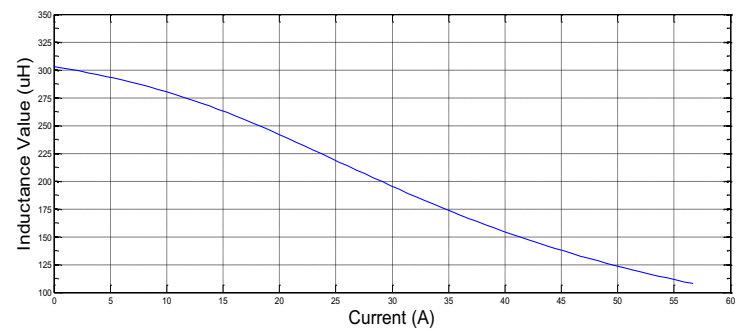

Figure 3. Decay curve of inductance

The coefficient is designed as follows: 


$$
\text { Kcomp }=L(i) / L_{0},
$$

Defining $L(i)$ the inductance value when current is $i$.

In the experience, a compensatory table should be firstly established. Then the new current controlled object can be improved to be as following:

$$
\begin{aligned}
& \operatorname{Gid}^{\prime}(s)=\operatorname{Gid}(s) * \operatorname{Kcomp} \\
& =\frac{V_{B U S}^{*}}{L(i) s} * \frac{L(i)}{L_{0}}=\frac{V_{B U S}^{*}}{L_{0} s}
\end{aligned}
$$

This is the final controlled object without decoupling the input voltage, bus voltage and even the variable inductance.

\section{Operation Result And Analysis}

\subsection{Introduced Experimental Platform}

In order to verify the performance of the control strategy, it was implemented on a $20-\mathrm{kva}$ ac/dc converter. It should be noted that, for such high-power applications, a three-phase boost converter is necessary to reduce the load of the converter on each phase. Fig. 4 shows the topology of the converter, and Fig. 5 shows the experimental prototype. The specifications of the converter are illustrated in Table 2.

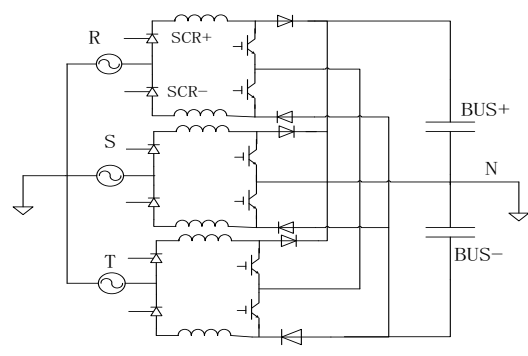

Figure 4. The Topology of the Converter

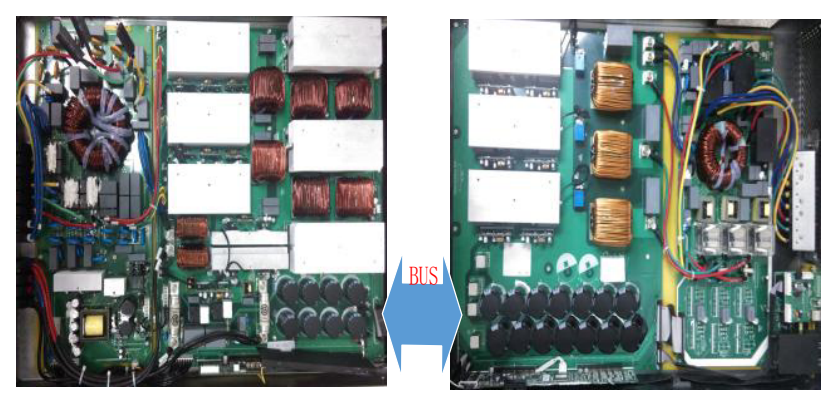

Figure 5. The Experimental Prototype

Table 2. Converter Specifications

\begin{tabular}{ccc}
\hline \hline Symbol & Parameter & Value \\
\hline$L$ & Boost PFC Inductor & $300 \mathrm{uH}$ \\
$C$ & BUS Capacitor & $8160 \mathrm{uF}$ \\
$V_{b u s}$ & BUS Voltage & $360 \mathrm{~V}$ \\
$P$ & Output Power & $18 \mathrm{KW}$ \\
$V_{\text {in }}$ & Grid Input Voltage & $176-264 \mathrm{~V} \mathrm{AC}$ \\
$\eta$ & Efficiency & 0.92 \\
$P F$ & Power Factor & $>0.98$ \\
$f_{s}$ & Switch Frequency & $19.2 \mathrm{KHz}$ \\
$f_{c n t}$ & System Clock Frequency & $150 \mathrm{MHz}$ \\
\hline \hline
\end{tabular}

\subsection{Dynamic Performance in Experiment}


Fig.6 shows the dynamics in Matlab Simulation. In the simulation, the technology of design based on the model has been used. The C-language program code which is running on the DSP has been imported into the Matlab C-Sfunction model. Therefore, the performance of the simulation is exactly the same as that of real experiment.

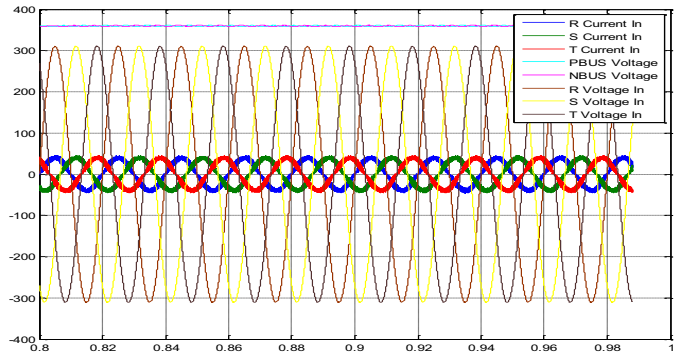

Figure 6. Matlab Simulation Result

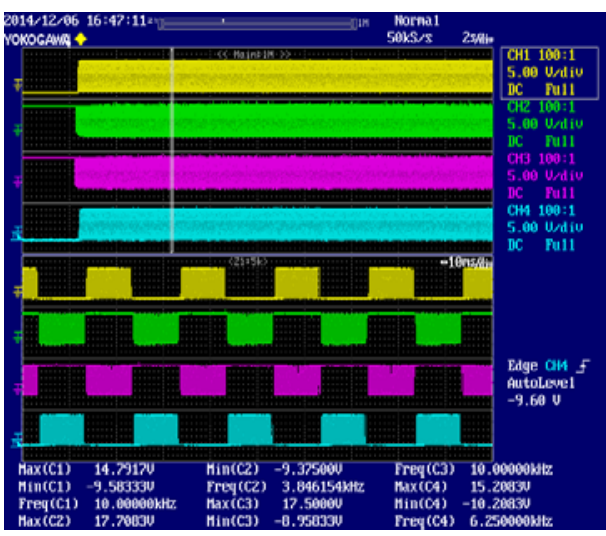

Figure 7. PWM duty-ratio of Phase R and Phase S

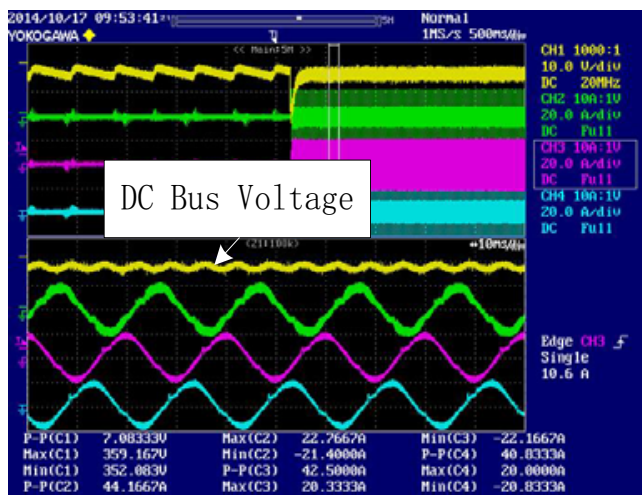

Figure 9. Response of the bus voltage for a $100 \%$ positive step load change

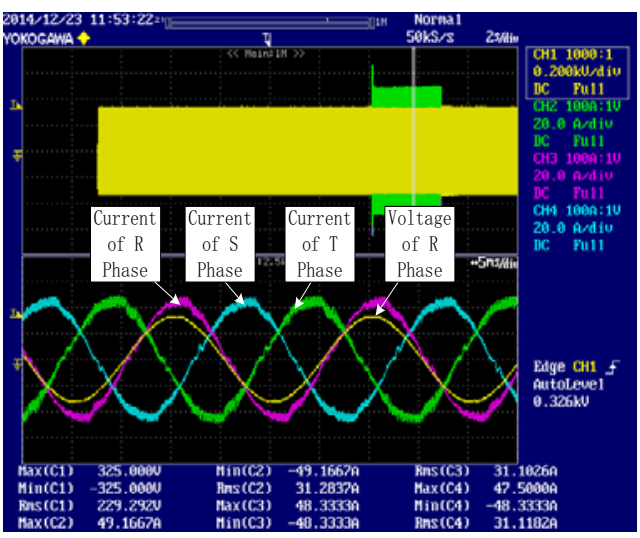

Figure 8. Three-phase input current and $\mathrm{R}$ phase input voltage

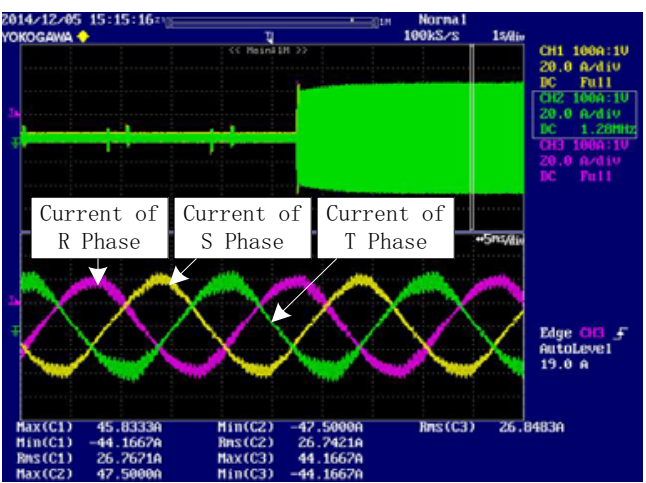

Figure 10. Three-phase input current without inductor compensation

Fig.7 shows the PWM duty-ratio of the converter. When the input voltage is positive, the positive boost works, so as the negative.

Fig. 8 shows the high performance of the PFC control. This figure confirms very good current quality, particularly for heavy loads. 
Fig.9 shows the transient response of the close-loop control system when a $100 \%$ step load change is applied to the converter. And it proves that the compensating coefficient and decoupling strategy is able to accurately maintain the stability of the DC bus.

Fig.10 shows that without the inductor compensation, the current fluctuates at its peak heavily. It is due to the variable inductance value.

\section{Conclusion}

This paper has presented a novel decoupling strategy for the boost PFC converter. This strategy has decoupled the input voltage and bus voltage, which make the system linear. Due to the proposed strategy, the PFC current is simply determined by the duty-ratio. Thus, the inner current controlled object has been simplified. In addition, a compensation coefficient is proposed to offset the inductance. It performs well not only in the simulation but also in the experiment.

\section{Acknowledgement}

This work was supported by National Natural Science Foundation of China under Grant 51475184.

\section{References}

1. L. Huber, B. T. Irving, M. M. Jovanović, "Closed-loop control methods for interleaved DCM/CCM boundary boost PFC converters,"Applied Power Electronics Conference and Exposition, 2009. APEC 2009. Twenty-Fourth Annual IEEE. IEEE, 2009 pp.991-997.

2. M. Pahlevaninezhad, S. Pan, S. Eren, et al, "An adaptive nonlinear current observer for boost PFC AC/DC converters".

3. R. B. Ridley, "Average small-signal analysis of the boost power factor correction circuit,"VPEC seminar proceedings. 1989 pp.108-120.

4. H. C. Chen, Z. H. Wu, Y. J. Liao,"Modeling and small-signal analysis of a switch-mode rectifier with single-loop current sensorless control," Power Electronics, IEEE Transactions on, 2010, 25(1) pp.75-84.

5. J. Sun,"On the zero-crossing distortion in single-phase PFC converters," Power Electronics, IEEE Transactions on, 2004, 19(3) pp.685-692.

6. M. Chen, J. Sun, "Feedforward current control of boost single-phase PFC converters,"Power Electronics, IEEE Transactions on, 2006, 21(2) pp. 338-345.

7. D. M. Van de Sype, K. De Gussemé, A. P. M. Van den Bossche, et al,"Duty-ratio feedforward for digitally controlled boost PFC converters,” Industrial Electronics, IEEE Transactions on, 2005, 52(1) pp.108-115.

8. J. Calvente, H. Ramirez-Murillo, E. Vidal-Idiarte, et al,"Multisampled average current control of switching power converters,"Industrial Technology (ICIT), 2015 IEEE International Conference on. IEEE, 2015 pp.2120-2124.

9. T. Nussbaumer, K. Raggl, and J. W. Kolar, "Design guidelines for interleaved single-phase boost PFC circuits,” IEEE Trans. Ind. Electron.,vol. 56, no. 7, pp. 2559-2573, Jul. 2009.

10. X. Lu, Y. X. Xie, L. Cheng, et al,"Semi-bridgeless boost PFC rectifier for wide voltage input range based on voltage feed-forward control,"Power Electronics and Motion Control Conference (IPEMC), 2012 7th International. IEEE, 2012, 3 pp.1894-1899.

11. F. Haase, A. Kouchaki, M. Nymand. "A low cost rapid prototype platform for a three phase PFC rectifier application,'Industrial Technology (ICIT), 2015 IEEE International Conference on. IEEE, 2015 pp.2024-2029. 